\title{
Perceptual Competition Promotes Suppression of Reward Salience in Behavioral Selection and Neural Representation
}

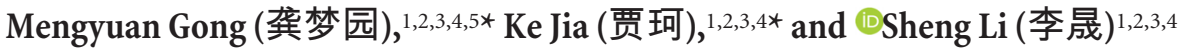 \\ ${ }^{1}$ School of Psychological and Cognitive Sciences, Peking University, Beijing, 100871, China, ${ }^{2}$ Beijing Key Laboratory of Behavior and Mental Health, \\ Peking University, Beijing, 100871, China, ${ }^{3}$ PKU-IDG/McGovern Institute for Brain Research, Peking University, Beijing, 100871, China, ${ }^{4}$ Key Laboratory of \\ Machine Perception (Ministry of Education), Peking University, Beijing, 100871, China, and 5 Department of Psychology, Michigan State University, \\ East Lansing, Michigan 48824
}

Visual attentional selection is influenced by the value of objects. Previous studies have demonstrated that reward-associated items lead to rapid distraction and associated behavioral costs, which are difficult to override with top-down control. However, it has not been determined whether a perceptually competitive environment could render the reward-driven distraction more susceptible to top-down suppression. Here, we trained both genders of human subjects to associate two orientations with high and low magnitudes of reward. After training, we collected fMRI data while the subjects performed a categorical visual search task. The item in the reward-associated orientation served as the distractor, and the relative physical salience between the target and distractor was carefully controlled to modulate the degree of perceptual competition. The behavioral results showed faster searches in the presence of high, relative to low, reward-associated distractors. However, this effect was evident only if the physical salience of the distractor was higher than that of the target, indicating a context-dependent suppression effect of reward salience that relied on high perceptual competition. By analyzing the fMRI data in primary visual cortex, we found that the behavioral pattern of results could be predicted by the suppressed channel responses tuned to the reward-associated orientation in the distractor location, accompanied by increased responses in the midbrain dopaminergic region. Our results suggest that the learned salience of a reward plays a flexible role in solving perceptual competition, enabling the neural system to adaptively modulate the perceptual representation for behavioral optimization.

Key words: fMRI; forward encoding model; perceptual competition; reward salience; suppression mechanism

\section{Significance Statement}

The predictiveness principle in learning theory suggests that the stimulus with high predictability of reward receives priority in attentional selection. This selection bias leads to difficulties in changing approach behaviors, and thus becomes an important factor related to psychiatric disorders with attentional deficits. Here, we demonstrated that such principle is adaptively implemented in attentional suppression in visual search. We showed that the learned salience induced the suppression of the rewardassociated distractor if its competition with the target was strong and could not be readily solved. This behavioral pattern was accompanied by increased midbrain fMRI activity and weakened sensory representation of the reward-associated distractor in V1. Our findings provided direct evidence that our brain flexibly uses learned regularities in attentional control.

\section{Introduction}

Competing for attentional priority has a vital role in sensory processing when the input information exceeds the limited ca-

Received Jan. 23, 2017; revised May 11, 2017; accepted May 15, 2017

Author contributions: M.G., K.J., and S.L. designed research; M.G. and K.J. performed research; M.G. and K.J. analyzed data; M.G., K.J., and S.L. wrote the paper.

This work was supported by the National Natural Science Foundation of China 31230029, 31470974, and 31271081.

The authors declare no competing financial interests.

*M.G. and K.J. contributed equally to this work.

Correspondence should be addressed to Dr. Sheng Li, School of Psychological and Cognitive Sciences, Peking

University, 5 Yiheyuan Road, Haidian, Beijing, 100871, China. E-mail: sli@pku.edu.cn. pacity of the sensory system (Motter, 1993; Desimone and Duncan, 1995; Ito and Gilbert, 1999; Kastner and Ungerleider, 2001; Fecteau and Munoz, 2006; Bisley and Goldberg, 2010; Ptak, 2012). This priority map can be biased by the selection of taskrelevant objects that is governed by top-down control, and the detection of unexpected or conspicuous objects in the form of bottom-up capture (Theeuwes, 1992; Egeth and Yantis, 1997).

Recent studies in both humans and primates have shown the advantage of reward-associated stimulus in attentional competi- 
tion when it is neither physically conspicuous nor task relevant (Della Libera and Chelazzi, 2009; Peck et al., 2009; Hickey et al., 2010; Anderson et al., 2011a). This learned predictiveness of reward was suggested to increase salience and upgrade its selection priority even when it was competing with a task-related and physically salient object (Hickey et al., 2010; Anderson et al., 2011a; Le Pelley et al., 2015). The evidence for the enhanced representation of reward-associated stimuli was found in both visual cortices (Serences, 2008) and dopaminergic midbrain areas (Anderson et al., 2014). Conversely, literature on cognitive control suggests that the dopaminergic modulation of sensory processing varied according to the task demands (van Schouwenburg et al., 2010). This high-level control theory predicts that the strength of dopaminergic modulation could also determine the degree of suppressed encoding of the reward-associated distractors (Hickey and Peelen, 2015). In agreement with this prediction, recent behavioral and electrophysiological investigations have demonstrated effective suppression over reward salience in certain task contexts (Lee and Shomstein, 2014; Sawaki et al., 2015; Gong et al., 2016). However, the mechanism of the suppression effect requires further investigation given the two streams of evidence in opposite directions.

Here, we suggest that an important step to reconcile the inconsistencies is to identify the critical factors that determine the suppression of reward salience in attentional competition. We believe that a plausible explanation concerning the controversy could be ascribed to the necessity of suppression in attentional control. If attending to the target is insufficient in solving perceptual competition, effective suppression of distractors with reward salience would become an alternative way to facilitate attentional selection. This idea is supported by the model of selective attention based on inhibition (Houghton and Tipper, 1994) and corresponding evidence, in which the strength of suppression was shown to adapt to the necessity of ignoring the distraction (Gaspar and McDonald, 2014; Weaver et al., 2017). To test this hypothesis, the experiment needs to meet two criteria. First, the strength of competition between the target and distractor needs to be carefully controlled: the physical salience of the target is either higher or lower than that of the distractor, thus creating conditions of low and high perceptual competition, respectively. Second, the degree of reward salience needs to vary between conditions only for the distractor. In this regard, the effect of reward salience on distractor suppression can be examined under different target-distractor relationships.

In the present study, we tested our hypothesis with a visual search task that met the above criteria. The behavioral results showed that the relative physical salience between the target and distractor played a critical role in the presence of suppression of reward salience. Particularly, the faster searches in presence of high, relative to low, reward associated distractors was evident only if the distractor's physical salience was higher than that of the target. Analyses of fMRI data revealed that this behavioral suppression effect under high competition was associated with increased activity in the midbrain area that is responsible for reward processing, as well as reduced orientation channel response in the area that responded to the reward-associated distractor in the primary visual cortex (V1).

\section{Materials and Methods}

\section{Experimental design and statistical analysis}

Participants. Thirty observers were recruited (14 males; $22 \pm 1.93$ years) for this experiment. They were students at Peking University with normal or corrected-to-normal vision, were right-handed, and gave written in- formed consent. The observers were paid $¥ 320$ ( $¥ 1 \approx \$ 0.15$ ) for completion of the tasks, with an additional payment up to $¥ 30$ based on their task performance in the reward learning session. The local ethics committee approved the study.

Procedure. Each observer performed three sessions of the main experiment over $3 \mathrm{~d}$. A practice session $(\sim 20 \mathrm{~min})$ was completed within 1 week before the main experiment. In the practice session, observers were shown the stimuli and trial sequences for each task, separately. After observers had familiarized themselves with the whole procedure and the tasks, they participated in three experimental sessions. (1) In the first scanning session $(\sim 1 \mathrm{~h})$, observers performed the orientation weight estimation task ( 6 runs; 72 trials/run; $380 \mathrm{~s} / \mathrm{run}$ ). (2) In the training session ( $\sim 50 \mathrm{~min})$, observers completed the reward-learning task (5 runs; 160 trials/run; 560 s/run). (3) In the second scanning session $(\sim 1 \mathrm{~h})$, observers performed the categorical visual search task ( 7 runs; 125 trials/ run; $270 \mathrm{~s} / \mathrm{run}$ ) and a separate run of retinotopic mapping (380 s). The practice and training session were completed in a behavioral laboratory, whereas the other two sessions were conducted at the MRI Research Center at Peking University.

Behavior design. The main experiment consisted of training (Session 2) and test (Session 3) sessions. We used the within-subject design for tasks in both sessions. Specifically, we first trained observers to associate two distinct orientations with high and low magnitudes of reward (Session 2), in which one independent factor of reward was manipulated. Then, we tested the suppression of reward salience with a visual search task (Session 3) in which the target was characterized by its category. In this categorical visual search task, one of these two reward-associated orientations served as a salient distractor, together with a salient target that had not been paired with reward before. Two independent factors (target's physical salience $X$ distractor's reward salience) were manipulated for the categorical visual search task. Each of the factors has two levels (high and low), producing four experimental conditions in total.

fMRI design. The fMRI data from the orientation weight estimation task (Session 1) were used to estimate the weights of the hypothesized orientation-tuned channels in each voxel for the forward encoding model (Brouwer and Heeger, 2009, 2011). Then, the forward encoding model was applied to collected fMRI data from V1 during the categorical visual search task (Session 3) to extract the voxels' responses at different orientation channels. This analytical approach has a major advantage in linking the changes in neural population activity directly to our manipulation of reward-associated orientations. We used the event-related design for tasks in both scanning sessions. In the orientation weight estimation task, each run contained balanced conditions of stimuli (eight orientations + fixation) and spatial locations (Fig. $1 \mathrm{~A}$, odd- or evennumbered locations). In the categorical visual search task, each run contained four experimental conditions and a fixation condition, with their presentation order arranged by $\mathrm{M}$-sequences (Buracas and Boynton, 2002) for each observer.

Statistical analysis. All statistical analyses on behavioral (i.e., search reaction time [RT] and accuracy) and neural indices (i.e., channel response and ROI-based activity) were performed using repeated-measures ANOVAs in SPSS 20.0 (IBM). The orientation selectivity for each voxel in V1 ROIs, as measured by ANOVA, were separately performed in MATLAB (The MathWorks; the resulting $F$ statistics were consistent with SPSS 20.0). Multiple comparisons were corrected for simple effects by using Bonferroni method to adjust the threshold of significance $(\alpha=0.025)$. The behaviorneural correlation analysis was performed using Pearson correlation, with two-tailed tests of significance.

\section{Stimuli and apparatus}

The stimuli were generated in MATLAB using Psychtoolbox. In the behavioral laboratory, the stimuli were displayed on a gray background of a gamma-corrected cathode ray tube (mean luminance: $22.5 \mathrm{~cd} / \mathrm{m}^{2}$ ) monitor (refresh rate: $60 \mathrm{~Hz}$; resolution: $1024 \times 768$ ). The viewing distance was $75 \mathrm{~cm}$. Observers pressed the buttons on the keyboard (left and right arrow keys) to make responses. Inside the MRI scanner, the stimuli were back-projected onto a translucent screen located inside the scanner bore (refresh rate: $60 \mathrm{~Hz}$; resolution: $1024 \times 768$ ). The observers viewed the stimuli at a distance of $75 \mathrm{~cm}$ through a mirror placed above their eyes. 


\section{A Orientation weight estimation task (session 1)}
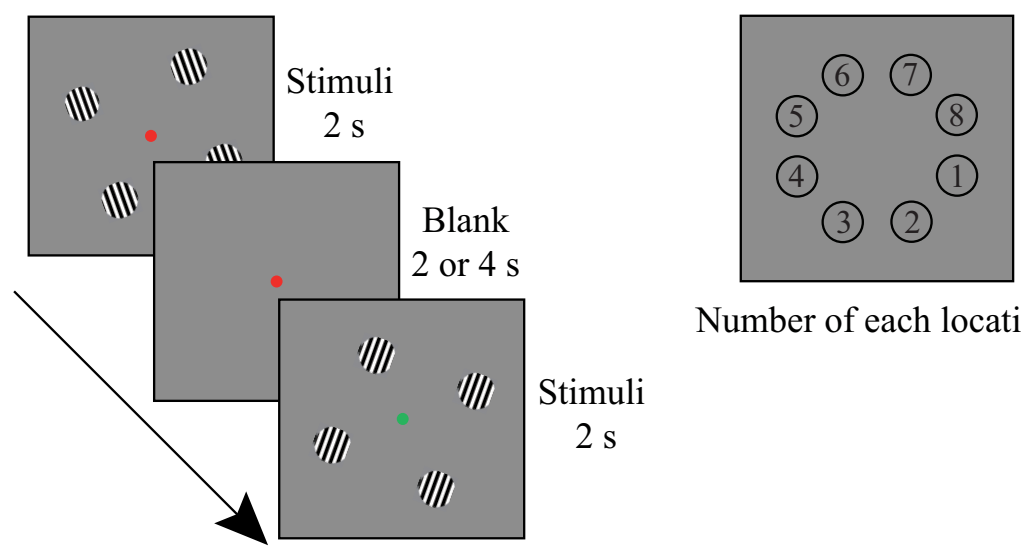

Number of each location
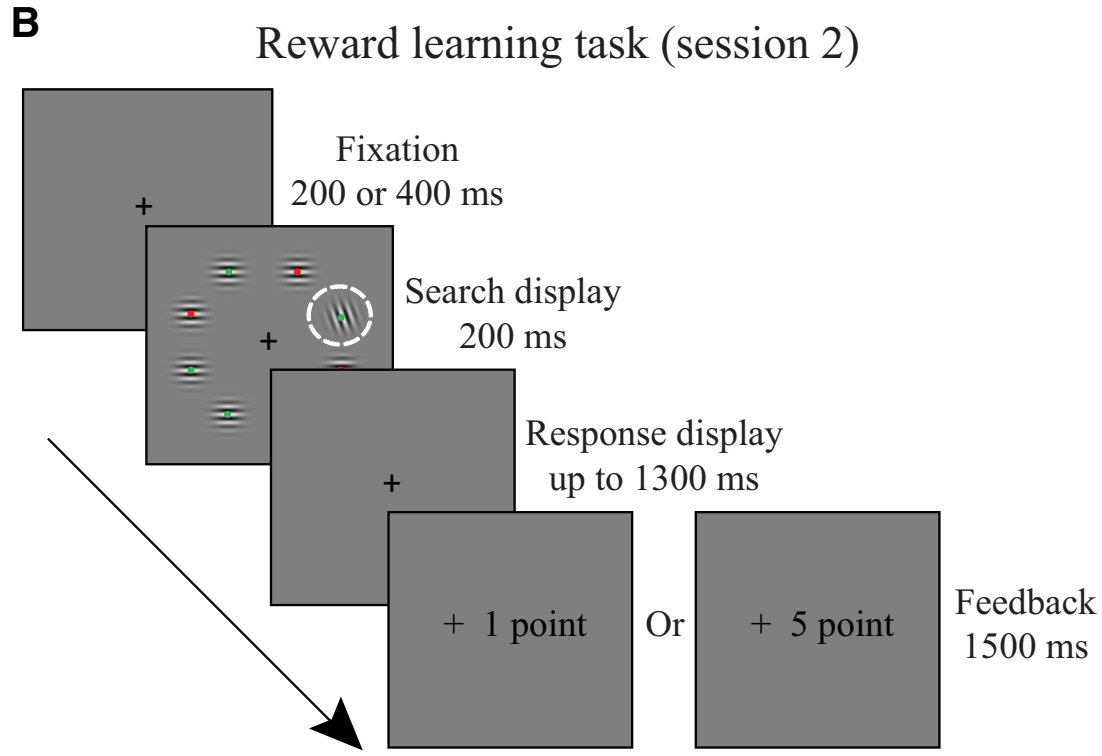

C
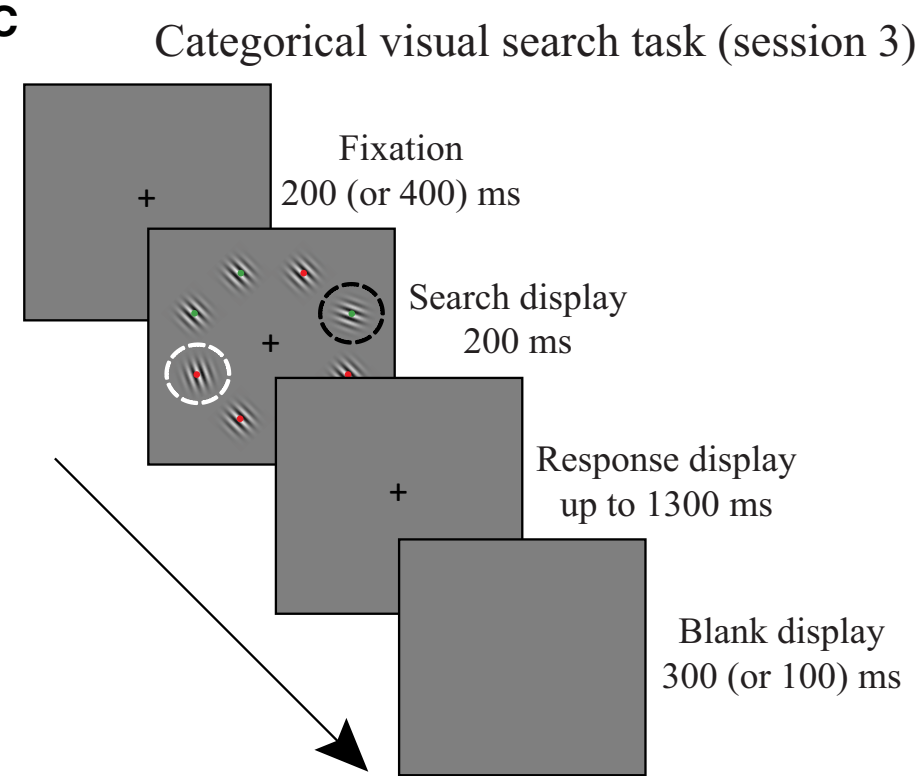

Figure 1. Experimental tasks. $\boldsymbol{A}$, Orientation weight estimation task (Session 1): observers performed a fixation task and detected the appearance of the green color (left), peripheral gratings were displayed in four of the eight possible positions (right). $\boldsymbol{B}$, Reward learning task (Session 2): observers performed a visual search task and reported the dot color inside a tilted Gabor. The
They were instructed to press the buttons on an MRI-compatible response box (left and right buttons).

As shown in Figure 2, half of the observers viewed a set of eight orientations (stimulus set 1: $\pm 20^{\circ}, \pm 45^{\circ}, \pm 60^{\circ}, \pm 80^{\circ}$ relative to vertical line), whereas the other half viewed a different set of orientations (stimulus set $2: \pm 10^{\circ}, \pm 30^{\circ}$, $\pm 45^{\circ}, \pm 70^{\circ}$ relative to vertical line). The motive for using two sets of stimuli was to balance the category of target (i.e., steep and shallow) in the training and test sessions. Notably, the search target in the training session reappeared as the distractor in the test session. The stimuli used in the training and test sessions were comprised of full-contrast Gabor patches (spatial frequency: 1.68 cycles/degree; SD: $0.54^{\circ}$; diameter: $\left.2.98^{\circ}\right)$. The full contrast-reversing sinusoid gratings (spatial frequency: 1.68 cycles/ degree; reverse rate: $4 \mathrm{~Hz}$; diameter: $\left.2.98^{\circ}\right)$ at different orientations (matched with the stimuli set for the training and the test session) were used in the orientation weight estimation task.

\section{Tasks}

Orientation weight estimation task (Session 1). Each run began and ended with an 8 and $12 \mathrm{~s}$ blank display, respectively. A static dot was shown at the center of the screen throughout the run. In each trial (Fig. 1A), four sinusoid gratings (diameter: $2.98^{\circ}$ ) of the same orientation were presented in four evenly spaced locations (Fig. 1A, odd- or even-numbered locations) for $2 \mathrm{~s}$, interleaved with an intertrial interval of 2 or $4 \mathrm{~s}$. The fixation dot flickered at a rate of $1 \mathrm{~Hz}$, with $90 \%$ probability of red color and $10 \%$ probability of green color. The observers were instructed to fixate on the central dot while passively viewing the gratings, and press a button with the index finger of their right hand whenever the dot was green.

Reward learning task (Session 2). As shown in Figure $1 B$, each trial started with a central fixation cross shown for 200 or $400 \mathrm{~ms}\left(0.6^{\circ} \times\right.$ $\left.0.6^{\circ}\right)$. Then, a search array composed of eight Gabor patches was presented around the fixation (eccentricity: $6^{\circ}$ ) with equal spacing for $200 \mathrm{~ms}$. The brief display was set to prevent eye movement. The target was a counterclockwise (CCW) or clockwise (CW) tilted Gabor singleton among the other seven horizontally or vertically oriented Gabors. Half of the observers were assigned to stimulus set 1 , in which the target orientation was $\pm 20^{\circ}$ relative to a vertical line (i.e., steep orientation). The other half of the observers were assigned to stimulus set 2 , in which the target orientation was $\pm 70^{\circ}$ relative to a vertical line (i.e., shallow orientation)

Gabor inside the white circle is the target. C, Categorical visual search task (Session 3): observers searched for a categorically defined Gabor (i.e., steep or shallow orientation) and reported the dot color inside the target. The Gabor inside the black circle is the target, and the Gabor inside the white circle is the distractor (the same orientation with the target in the reward learning task). The dashed circles were not visible in the experiment. 
Search target

Stimulus set

Training task

Test task

\begin{tabular}{ccc}
\hline Set 1 & Steep orientation & Shallow orientation \\
(i.e., $\left.\pm 20^{\circ}\right)$ & (i.e., $\pm 60^{\circ}$ or $\pm 80^{\circ}$ ) \\
high reward salience \\
low reward salience
\end{tabular}

Figure 2. The two stimulus sets used in the experiment and the definition of the search targets in the training session (Session 2) and test session (Session 3) for each stimulus set.

(Fig. 2). A red or green dot was randomly placed at the center of each Gabor (four red dots and four green dots in each display). The observers were asked to identify the dot color in the target Gabor by pressing a button with their right index and middle fingers. After the offset of the search array, a blank screen was shown for $1300 \mathrm{~ms}$ (or until response). Correct response was followed by a high ( +5 points) or low reward $(+1$ point) feedback displayed for $1500 \mathrm{~ms}$. The CCW-and CW-tilted Gabors were shown with equal probability and were, respectively, associated with a high probability $(80 \%)$ of a high reward and low reward for half the observers, and vice versa for the other half. The total reward earnings were converted to extra payment at the end of the experiment. All observers were naive to the reward-orientation contingency, and most of them reported being unaware of such contingency after completing the whole experiment.

Categorical visual search task (Session 3). Each run began and ended with an 8 and $12 \mathrm{~s}$ blank display, respectively. A static red dot was shown at the center of the screen in the blank display. As shown in Figure $1 C$, each trial started with a central fixation cross $\left(0.6^{\circ} \times 0.6^{\circ}\right)$ shown for 200 (or $400 \mathrm{~ms}$ ). A search array composed of eight tilted Gabors was presented around the fixation (eccentricity: $6^{\circ}$ ) with equal spacing for $200 \mathrm{~ms}$. The eight Gabor items were always of the same titled direction (i.e., either $\mathrm{CCW}$ or $\mathrm{CW}$ ). Each array consisted of a
Gabor in the target orientation and a distractor Gabor in a previously rewarded orientation; the other Gabors were of the same diagonal orientation (i.e., CCW or CW $45^{\circ}$ ) serving as the background stimuli. The observers were assigned to stimulus set 1 or 2 to search for a shallow orientation $\left( \pm 60^{\circ}\right.$ or $\pm 80^{\circ}$ relative to vertical line) or a steep orientation $\left( \pm 10^{\circ}\right.$ or $\pm 30^{\circ}$ relative to vertical line $)$, respectively. The target salience was determined solely by its physical salience that depended on the orientation contrast between the target and the background (i.e., $15^{\circ}$ or $35^{\circ}$ ). Therefore, it could either be higher or be lower than the physical salience of the distractor (i.e., $25^{\circ}$ ). On the other hand, the distractor salience was jointly determined by the physical salience and its associated magnitude of reward. Particularly, we defined the target and distractor using the same feature dimension (i.e., orientation) to compensate for the uncontrollable low-level perceptual differences when the physical salience of the target and distractor was manipulated in different feature dimensions (e.g., color and shape). The observers were instructed to discriminate the dot color in the target Gabor, which was the same as in the reward learning task. After the offset of the search arrays, a fixation display was shown for $1300 \mathrm{~ms}$ to wait for a button press followed by a blank display of $300 \mathrm{~ms}$ (or $100 \mathrm{~ms}$ ). No feedback was given in this task. 


\section{MRI data acquisition}

Imaging data were collected from a Siemens 3T Prisma scanner, equipped with a 64-channel head coil at Peking University. For each observer and each scanning session, high-resolution anatomical images were acquired using MPRAGE T1-weighted sequence (FOV: $256 \times 224$ $\mathrm{mm}$; resolution: $0.5 \times 0.5 \times 1 \mathrm{~mm}$; number of slices: 192$)$. Functional images were acquired using EPI sequence (TR: $2000 \mathrm{~ms}$; TE: $30 \mathrm{~ms}$; FOV: $224 \times 224 \mathrm{~mm}$; flip angle: $90^{\circ}$; matrix size: $64 \times 64$; resolution: $3.5 \times$ $3.5 \times 3.5 \mathrm{~mm}$, gap: $0.7 \mathrm{~mm}$; number of slices $=33$ ).

\section{fMRI data preprocessing}

Imaging data were processed using BrainVoyager QX (Brain Innovation) software. Preprocessing of functional data included slice-timing correction, motion correction, temporal filtering ( 3 cycles), and removal of linear trends. Spatial smoothing (4 mm Gaussian filter, FWHM) was only applied to the region of interest (ROI) analysis. For each scanning session, the functional data were first aligned to their first functional slice and then coregistered to the anatomical data obtained in the same session. The anatomical images in the second session were aligned to those in the first session. For the group-level analysis, the aligned functional data were resampled into $3 \times 3 \times 3 \mathrm{~mm}$ resolution and transformed into the standardized Talairach space. Trials with incorrect responses in the categorical visual search task were eliminated from further analysis.

\section{Forward encoding model}

The forward encoding model allows us to infer how feature-specific neuronal populations are modulated by experimental manipulation, rather than the pooled activity across orientations at the voxel level (Serences and Saproo, 2012). The model assumes that each voxel contains a large number of orientation selective neurons, and the orientation selectivity of each neuron can be characterized as a linearly weighted sum of eight hypothetical orientation channels (Brouwer and Heeger, 2009, 2011). Each channel is represented by a basis function that models its idealized orientation tuning curve. In practice, each basis function is a sinusoid raised to the sixth power to approximate the tuning function of V1 neurons (Schiller et al., 1976; Swindale, 1998), with the orientation preference selected from one of the stimulus sets. The reconstruction of channel responses followed three stages. First, we defined the ROIs in V1 that corresponded to the eight locations in the search array. Second, we estimated the channel weights for each voxel within the ROI. Third, we calculated the channel responses associated with each of the orientations in the stimulus set.

ROI definition. For each observer, we defined the location-specific V1 ROIs by selecting the voxels in V1 that responded to the stimulation at each of the eight locations using data from retinotopic mapping and orientation weight estimation runs (Fig. $3 A$ ). To define the V1 boundary, we used the standard phase-encoded method (Sereno et al., 1995; Engel et al., 1997) in which observers viewed rotating wedge that created traveling waves of neural activity in the visual cortex. To identify eight corresponding locations within V1, we applied a GLM to the data collected during the orientation weight estimation runs (Session 1). There were two regressors in the GLM that corresponded to two conditions where the stimuli were displayed at odd- or even-numbered locations. We mapped separate voxel neighborhoods in V1 that were significantly modulated by one of the two conditions as the location-specific ROIs.

Channel weights. For each observer, we used functional data collected during the orientation weight estimation runs (Session 1) to compute the channel weights for the forward encoding model. First, we estimated the hemodynamic impulse response functions (HIRFs) for each voxel in the ROIs with the deconvolution method (Dale, 1999) (Fig. 3B). The design matrix consisted of two conditions of the stimulus location (i.e., odd- or even-numbered locations) concatenated across runs, regardless of the orientations. HIRFs were calculated by multiplying the pseudoinverse of this design matrix with the preprocessed fMRI response time courses. Next, we estimated the response amplitudes $(\beta)$ to each orientation separately for each voxel and each run. The regression matrices were constructed by convolving the location-specific HIRF with a binary time course corresponding to the onset of each of the eight orientations. The response amplitudes were calculated by multiplying the pseudoin- verse of the regression matrices with the preprocessed fMRI response time courses, and then normalized ( $z$-score) separately for each voxel and for each run. To enhance the signal-to-noise ratio, we selected a subset of informative voxels that responded most differentially across orientations (Brouwer and Heeger, 2011). For each voxel, we performed an ANOVA on its normalized responses across eight orientations, with the number of runs as the repeated measurements. Each voxel's orientation selectivity can be denoted by $F$ statistics (i.e., a larger $F$-statistic indicates higher selectivity). Because of the small physical size of each location in visual space, we were able to identify only a small neighborhood of voxels in V1 for each location. We selected 30 voxels (i.e., the minimal amount across ROIs and observers) with the largest $F$ statistics for further analysis (Fig. $3 C$ ), controlling for possible differences in variation in the number of voxels.

Let $m$ be the number of selected voxels (i.e., 30), $n_{1}$ be the number of repeated measurements (i.e., 8 orientations $\times 6$ runs), and $k$ be the number of hypothetical channels. As shown in Figure $3 D$, we constructed the matrix of selected voxel responses that corresponded to each of the orientations in the stimulus set $\left(B_{1}, m \times n_{1}\right)$ (response matrix). The orientation tuning model was comprised of the matrix associated with the hypothetical channel responses $\left(C_{1}, k \times n_{1}\right)$. The least-squares estimates were used to compute the channel weights matrix $(W, m \times k)$ as follows:

$$
\begin{gathered}
B_{1}=W C_{1} \\
\hat{W}=B_{1} C_{1}^{T}\left(C_{1} C_{1}^{T}\right)^{-1}
\end{gathered}
$$

Channel responses. For each observer, we used functional data from the categorical visual search task (Session 3) to compute the channel responses. For each trial, we obtained the response amplitudes from the selected 30 voxels in ROIs corresponding to the target- and distractorrelated locations separately. We constructed a matrix of voxel response by extracting the peak amplitude of each voxel ( $6 \mathrm{~s}$ after the stimulus onset, see Fig. $3 B$ ), using their coordinates in normalized space. Let $n_{2}$ be the number of trials, and the channel responses $\left(C_{2}, k \times n_{2}\right)$ associated with the distributed pattern of activity during the categorical visual search task were estimated based on the matrix of voxel responses $\left(B_{2}\right.$, $m \times n_{2}$ ) (Fig. $3 D$, voxel response) and the weights matrix acquired from the first stage of analysis. After this process, we separately grouped the responses of channels preferring the target and distractor orientations after sorting the trials into four experimental conditions as follows:

$$
\hat{C_{2}}=\left(\hat{W}^{T} \hat{W}\right)^{-1} \hat{W}^{T} B_{2}
$$

\section{Midbrain ROI analysis}

To test the possible role of dopaminergic modulation in the categorical visual search task (Session 3), we drew the ventral tegmental area (VTA) on the normalized high-resolution MR image for each observer by hand. Using Mango image processing software (http://ric. uthscsa.edu/mango/), we drew the VTA in the axial section (Fig. 4E, left) following the descriptions of boundaries by Ballard et al. (2011) and created volumes of interest. The mean volume of the VTA across observers was $156 \pm 19.7 \mathrm{~mm}^{3}$. The mean coordinates for the VTA in MNI/Talairach space were as follows: (left) $[-2.0,-18.2,-14.5] /$ $[-2.1,-18.2,-10.4]$ and (right) $[2.4,-18.8,-14.3] /[1.7,-18.2$, $-10.7]$. The estimated volume and coordinates were similar to previously reported results (Eapen et al., 2011; Murty et al., 2014).

\section{Results}

\section{Behavioral results}

During the reward learning task, observers' performance improved over time for both the high and low reward conditions (Fig. 4A). We grouped the data from this task into 5 time bins, one for each run. The repeated-measures ANOVA (reward condition $\times$ time bin) revealed a main effect of time bin for search accuracy $\left(F_{(4,116)}=23.23, p<0.001, \eta_{p}^{2}=0.45\right)$ and a tendency for faster RTs $\left(F_{(4,116)}=2.19, p=0.074, \eta_{p}^{2}=0.07\right)$. These results indicated the observers' active involvement in the task. 
A

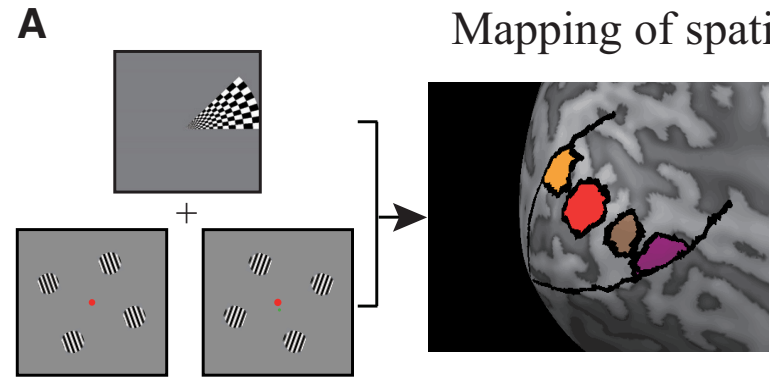

Mapping of spatial locations in V1

B

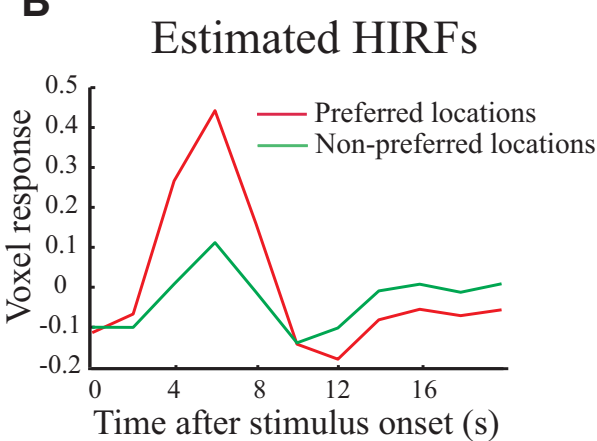

C

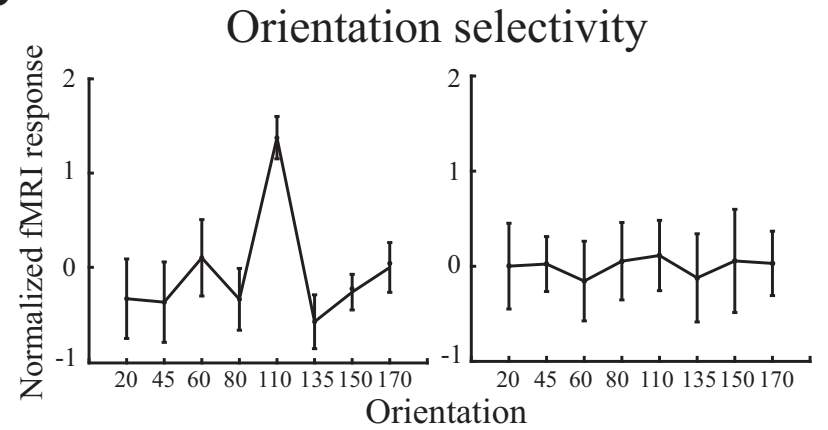

D

Orientation tuning model

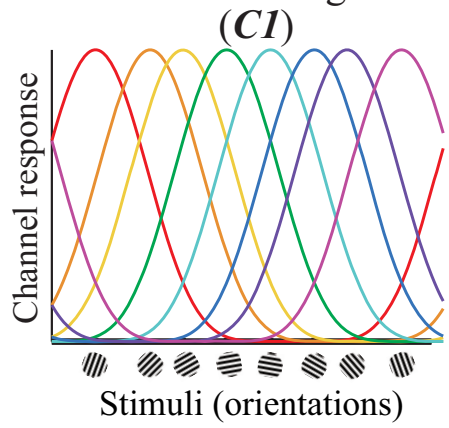

Session 1: response matrix (B1)

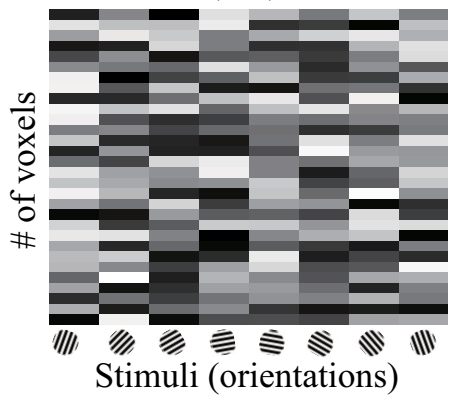

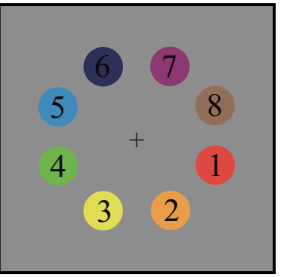

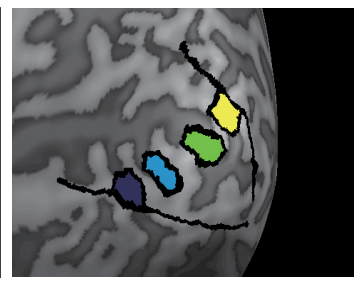

Channel responses

(C2)
Target ROI
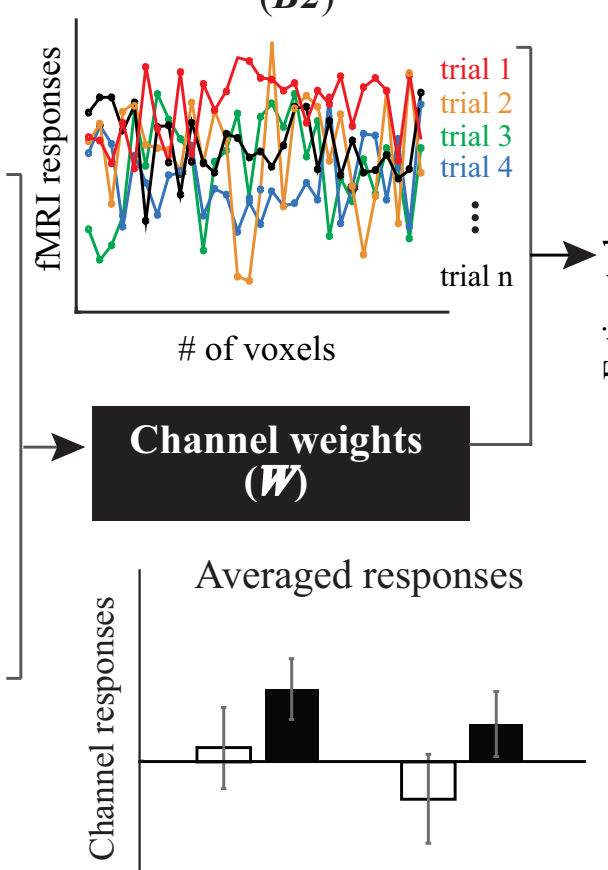

Experimental conditions

Figure 3. Schematic illustration of fMRI data analysis. $A$, An example of hand-drawn location-specific ROls in V1. The eight locations corresponding to the search arrays were determined by combining the activations from the retinotopic mapping and orientation weight estimation tasks. $B$, Example of the estimated HIRFs corresponding to the voxel's preferred and nonpreferred spatial locations. C, Two representative voxel responses across orientations ( $z$-normalized). The degree of orientation selectivity for each voxel was characterized using $F$ statistics. The voxels on the left and right sides exhibited high and low orientation selectivity, respectively. $\boldsymbol{D}$, Two stages of the forward encoding model (left to right). Channel weights estimation: the response of each voxel was modeled as a weighted sum of eight orientation channels, and each channel corresponded to one orientation in the stimulus set (orientation tuning model, ( 1 ). With the response amplitudes for each voxel across orientations in multiple runs (Session 1: response matrix, $\boldsymbol{B}$ 1), we estimated the weight of each channel $(\boldsymbol{W})$ to each voxel using Equation 2. Channel response reconstruction: the estimation of channel weights for each voxel and preprocessed fMRI signal across voxels (Session 3: voxel responses, B2) in each trial enabled us to reconstruct the channel response (C2) in specific ROls using Equation 3. The resulting channel responses were separately grouped after sorting the trials into four experimental conditions. 
A
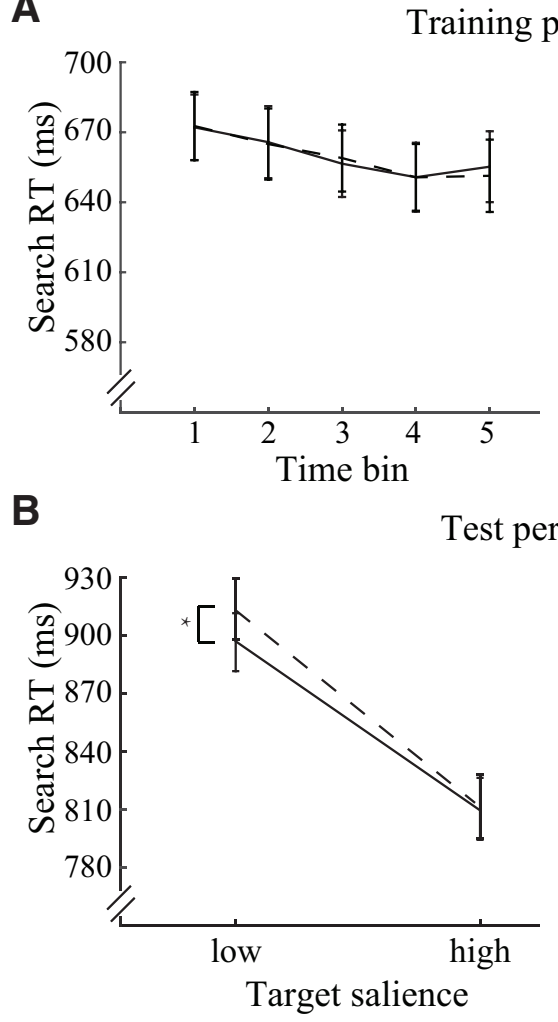

C

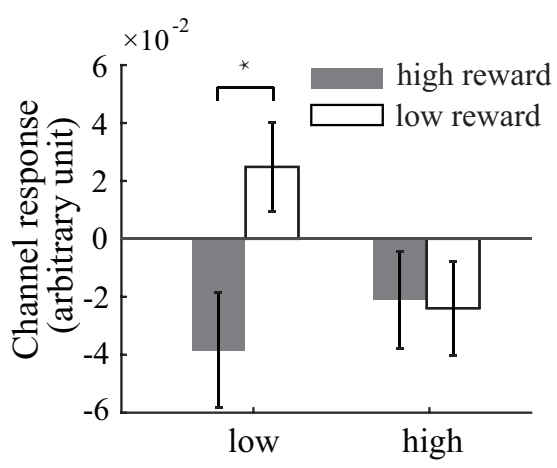

Target salience
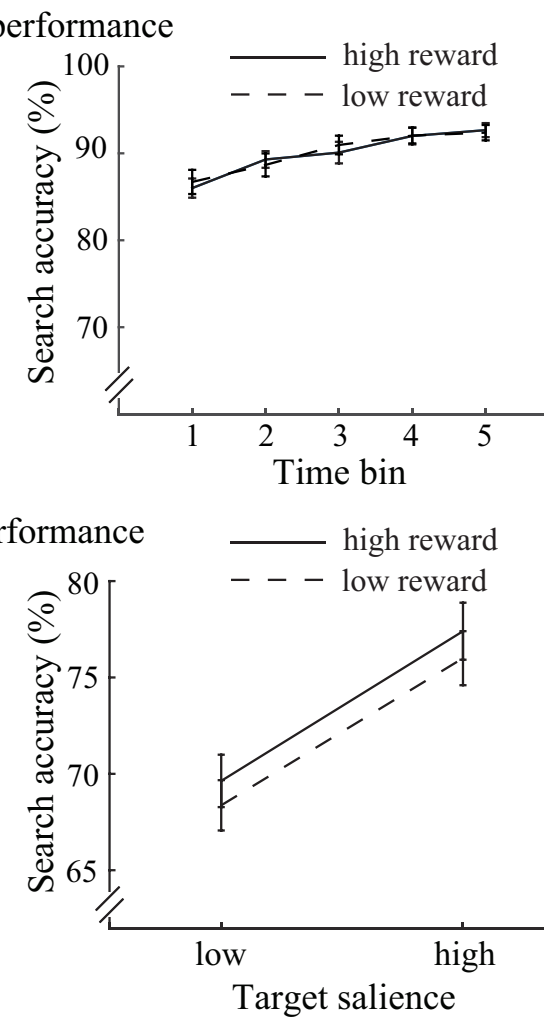

D Low salience target condition

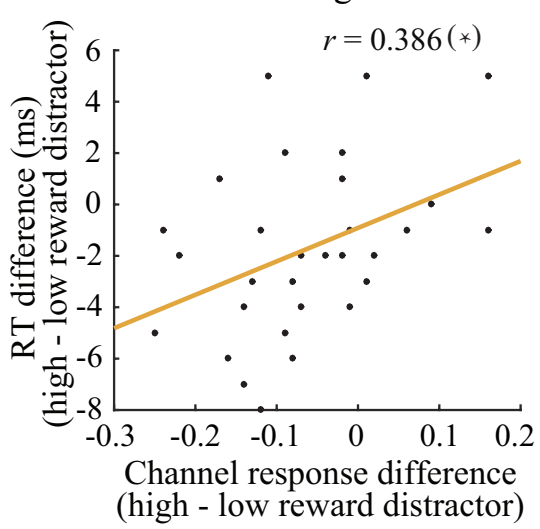

E

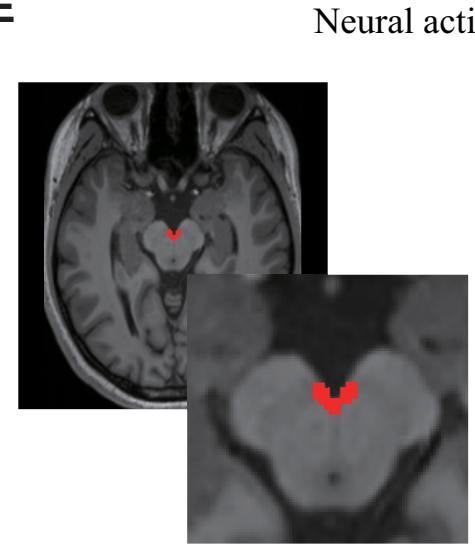

Neural activation in VTA

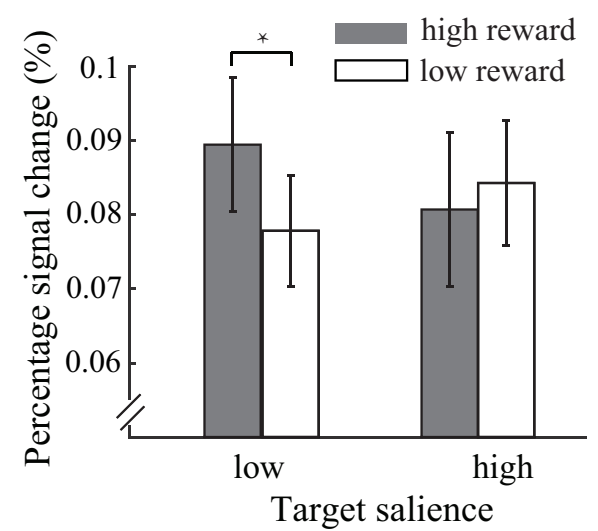

Figure 4. Behavioral results and fMRI results. $\boldsymbol{A}$, Search RT and accuracy in the reward learning task. $\boldsymbol{B}$, Search RT and accuracy in the categorical visual search task. C, Distractor-related channel responses in distractor ROIs across experimental conditions. D. Correlation between the reward effect on RT and distractor-related channel response in distractor ROls in V1.
However, the influence of reward was not shown either as a main effect $(p>0.6$ for search accuracy and RTs) or an interaction effect $(p>0.8$ for search accuracy and RTs). Studies that used similar reward associative learning paradigms have shown mixed results regarding the reward effect during training. The influence of reward could be observed on the mean training performance (Sha and Jiang, 2016; Anderson et al., 2016), in the last block of training (Anderson et al., 2014), or on the second day of training (Laurent et al., 2015), whereas similar paradigms could also lead to nonreward effects during training (Anderson et al., 2011b; Chelazzi et al., 2014; Gong and Li, 2014). Despite the large variance in reward effects during training across studies or within the same study across experiments (Gong et al., 2016; Rajsic et al., 2017), all of these studies reported findings of significant reward effects on multiple test tasks.

Although the effect of reward was not shown during training, its impact on the test session confirmed the observers' acquisition of reward-orientation associations. The mean behavioral accuracy in the categorical visual search task was $72.8 \%$, confirming that the task had an intermediate level of difficulty. Figure $4 B$ (left) shows the search RTs of the correct trials during the categorical visual search task. We entered the RT data into a repeatedmeasures ANOVA (target's physical salience $X$ distractor's reward salience). The main effects of reward salience $\left(F_{(1,29)}=\right.$ $\left.4.45, p=0.044, \eta_{p}^{2}=0.13\right)$ and physical salience $\left(F_{(1,29)}=185.49, p<0.001\right.$, $\left.\eta_{p}^{2}=0.86\right)$ were significant. Additionally, the two-factor interaction was marginally significant $\left(F_{(1,29)}=4.16, p=\right.$ $\left.0.051, \eta_{p}^{2}=0.13\right)$. Simple effect analysis suggests that the suppression of the reward-associated distractor was critically dependent on the physical salience of the target (low salience: $F_{(1,29)}=8.66, p=$ $0.006, \eta_{p}^{2}=0.23$; high salience: $F_{(1,29)}=$ $0.09, p=0.769, \eta_{p}^{2}=0$; Bonferronicorrected threshold: $\alpha=0.025)$. That is, when the target was physically less salient than the distractor $\left(15^{\circ}\right.$ vs $\left.25^{\circ}\right)$, there was stronger suppression over the distractor with high reward salience. On the contrary, when the target was physically more

\footnotetext{
$\boldsymbol{E}$, Example of a hand-drawn ROl for the VTA in a highresolution MR image (left) and the results of the percentage signal change analysis of the VTA during the categorical visual search task (right). *Significant differences.
} 
salient than the distractor $\left(35^{\circ}\right.$ vs $\left.25^{\circ}\right)$, the search RTs of the trials with high- and low-reward associated distractors were indistinguishable. This finding is consistent with our hypothesis that suppression of a distractor with reward salience occurs if attending to the target is insufficient for solving perceptual competition. On the other hand, the effect induced by target-related physical salience was independent of the distractor's reward salience (low salience: $F_{(1,29)}=141.79, p<0.001, \eta_{p}^{2}=0.83$; high salience: $F_{(1,29)}=143.74, p<0.001, \eta_{p}^{2}=0.83$; Bonferroni-corrected threshold: $\alpha=0.025$ ).

A repeated-measures ANOVA was also used to examine search accuracy (Fig. $4 B$, right). The results showed a main effect of reward salience $\left(F_{(1,29)}=4.67, p=0.039, \eta_{p}^{2}=0.14\right)$ and physical salience $\left(F_{(1,29)}=49.40, p<0.001, \eta_{p}^{2}=0.63\right)$. No interaction was found between the two factors $\left(F_{(1,29)}=0.004, p=\right.$ $\left.0.949, \eta_{p}^{2}=0\right)$. These results demonstrated that the behavioral benefits related to our reward manipulation were not due to a speed-accuracy tradeoff. The interaction effect in RT also ruled out the possibility of an arousal effect induced by the appearance of high reward-associated distractors, allowing the behavioral data to be best accounted for by the suppression of the rewardassociated distractor.

\section{Forward encoding model: the suppressed channel response toward a reward-associated distractor}

We were motivated to use the forward encoding model for two reasons. First, each voxel contained neurons that were tuned to a wide range of orientations, whereas our reward manipulation was only related to two particular orientations. Examining the channel-based signal change at the neuronal population level improves the signalto-noise ratio compared with voxel-level analysis. Second, the suppression of the reward-associated orientation might, at the same time, decrease the response of the neurons that were tuned to that orientation and increase the response of the neurons that were tuned to other orientations (Martinez-Trujillo and Treue, 2004). These response changes may cancel each other out and lead to negligible overall changes at the voxel level.

For each observer and each trial, we separately calculated the channel responses in the ROIs corresponding to the locations of the target and distractor. The averaged channel responses for each experimental condition were computed and entered into repeated-measures ANOVAs (target's physical salience $\times$ distractor's reward salience). In the distractor-related ROI (Fig. 4C), we found that the channel preferring the distractor orientation showed a significant main effect of reward salience $\left(F_{(1,29)}=4.77\right.$, $\left.p=0.037, \eta_{p}^{2}=0.14\right)$ and a significant interaction between the two factors $\left(F_{(1,29)}=7.87, p=0.009, \eta_{p}^{2}=0.21\right)$, whereas the main effect of physical salience did not reach significance $\left(F_{(1,29)}=1.13, p=0.296, \eta_{p}^{2}=0.04\right)$. Simple effect analysis revealed a reward-driven reduction of channel responses when the distractor was physically more salient than the target, but not vice versa (low target salience: $F_{(1,29)}=11.67, p=0.002$, $\eta_{p}^{2}=0.29$; high target salience: $F_{(1,29)}=0.03, p=0.873$, $\eta_{p}^{2}=0.001$; Bonferroni-corrected threshold: $\left.\alpha=0.025\right)$. The sign of the channel response values in V1 (Fig. 4C) could not provide direct indication for either enhanced or suppressed responses of specific channels due to the normalization of neural responses in the data preprocessing. The relative values between the two levels of reward condition were of the interest for these channel responses. These results suggest that the two factors (i.e., physical salience and reward salience) interacted to jointly determine the outcome of attentional competition. For the channel that preferred the target orientation, we found no significant main effect of reward salience or two-factor interaction, and only a marginally significant effect of physical salience $\left(F_{(1,29)}=3.84\right.$, $p=0.060, \eta_{p}^{2}=0.12$ ).

These results are well aligned with the explanation for behavioral performance in the categorical visual search task, indicating a potential correlation between behavioral effects and the effects in channel responses. To test this possibility, we calculated the differences between two levels of reward condition (i.e., high vs low distractor's reward salience) separately for the search RT and distractor-related channel responses under two levels of physical salience condition (i.e., low and high target's physical salience). A significant correlation was found in the low target salience condition ( $r=0.386, p=0.035$, two-tailed Pearson correlation; Fig. $4 D$ ), whereas such correlation was not shown in the high target salience condition $(r=-0.26, p=0.165$, two-tailed Pearson correlation).

Similar repeated-measures ANOVAs were conducted on the channel responses in the target-related ROIs. There were no effects of channel responses that were tuned to the target and distractor orientations (for all comparisons, $p>0.16$ ). To confirm the specificity of the response reduction for the distractor orientation, we analyzed the channel responses for neutral distractors in the search arrays (i.e., CCW and CW $45^{\circ}$ ) separately in the target- and distractor-related ROIs. None of these channels showed significant differences across experimental conditions (for all comparisons, $p>0.11$ ).

Together, the reward-induced reduction of channel response was specific to the high reward-associated orientation channel in the distractor-responsive location in V1. The changes in channel responses can be seen as the neuronal solution to weaken the competition of the reward-associated distractor in a very early stage of visual processing. The distractor-related channel responses that covaried with behavioral performance reflected the modulation of reward depending on the target-distractor competition. Importantly, the outcome of the competition was demonstrated with the significant interaction effect of the channel responses. Specifically, in the high target salience condition, the target was likely to dominate the competition with the distractor because it was favored by both the task goal and physical salience. Therefore, regardless of the reward salience, the representation of the distractor was inevitably weakened under this condition, as can be observed in the behavioral results in Figure $4 B$ (faster RTs) and channel responses in Figure $4 C$ (smaller responses). On the other hand, when the target was only favored by the task goal in the low target salience condition, the representation of the distractor was supposed to be higher due to its high physical salience under this condition. This difference may contribute to the higher response for the low reward distractor in the low target salience condition. Meanwhile, there was better suppression of the high-reward associated distractor that reduced its channel response to a level that was similar to the high target salience condition. These results suggest that the representation of the distractor can be easily overridden when the target was physically more salient, whereas active suppression over distractors occurred when the distractor was more salient in terms of physical salience and reward association.

\section{ROI analysis: activation in the midbrain dopaminergic area}

To test whether dopaminergic modulation contributes to the observed reward effect, we compared the VTA activity across experimental conditions. Consistent with the pattern of results in behavior and forward encoding models (Fig. 4E), we found a significant interaction effect of VTA activity in its peak response (i.e., 
$4 \mathrm{~s}$ after stimulus onset): $\left(F_{(1,29)}=5.73, p=0.023, \eta_{p}^{2}=0.17\right)$. Further simple effect analysis revealed a reward-enhanced response depending on the salience of the target (low target salience: $F_{(1,29)}=$ $6.75, p=0.015, \eta_{p}^{2}=0.19$; high target salience: $F_{(1,29)}=0.46, p=$ $0.503, \eta_{p}^{2}=0.02$; Bonferroni-corrected threshold: $\left.\alpha=0.025\right)$. This result implies that the midbrain activity related to reward salience contributed to the behavioral suppression effect in the low target salience condition.

\section{Discussion}

The present study provided direct evidence that can account for the contradictory results in the literature regarding the suppression of reward salience. Our results showed that the suppression of a reward-salient distractor was evident if its physical salience was higher than that of the target, indicating the important role of target-distractor competition in the suppression mechanism. Critically, the high reward-salient distractor was better suppressed in the low target salience condition, probably because the top-down target selection was not sufficient to win the perceptual competition easily. This behavioral suppression effect was accompanied by weakened competition from the neuronal population in V1 that responded to the distractor with the high reward-associated orientation. On the other hand, suppression of reward salience was not observed when it was unnecessary in the high target salience condition, in which the target dominated the competition with the distractor and was favored by both topdown and bottom-up attentional selection.

Our results demonstrate that, when the distinction between the target and distractor is unambiguous (e.g., categorically separable), top-down suppression of distractors can take place if necessary. These findings agree with current literature in two ways. First, the results are well aligned with the attention model based on inhibition that suggests stronger suppression for more potent distractors (Houghton and Tipper, 1994; Houghton et al., 1996). The recent electrophysiological results have demonstrated that this is indeed a possible mechanism used by the human brain to facilitate visual search performance when a distractor is highly salient (Gaspar and McDonald, 2014; Gaspar et al., 2016; Weaver et al., 2017). Second, the results dovetail with the recent proposal of the signal suppression hypothesis of controlled attentional capture (Sawaki and Luck, 2010, 2011; Sawaki et al., 2012). This theory emphasizes the situation where the physically salient item could be better suppressed due to the active suppression of salience-induced attentional priority (Geng and Diquattro, 2010; Gaspelin et al., 2015), the rationale of the reversed salience effect is the dominant control of top-down attention. In our study, the observers were aware of the attribute of the target (e.g., the steep orientation) and the to-be-ignored distractor (e.g., the shallow orientation), making the top-down control ready to initiate the suppression mechanism. However, an initial attraction to the distractor with high reward salience followed by strong suppression could be an alternative mechanism for our findings, as indicated by a study using time-sensitive measures of eye movement (Hickey and van Zoest, 2012). Notably, the suppression of reward salience reduced its impairment on performance did not eliminate the physical salience effect on the search performance. It was the task goal, physical salience, and the reward history that worked together to modify the priority map for attentional selection (Awh et al., 2012).

Apart from the theoretical implications for the suppression of reward salience, our results can also be reconciled with the empirical studies that did not observe this suppression effect (Hickey et al., 2011; Anderson et al., 2014; Failing et al., 2015; Le Pelley et al., 2015). Particularly, the search target in these test tasks was always defined by feature singleton (i.e., search for a unique shape), allowing visual search to be dominated by the singleton detection mode. In contrast to these studies where there was not a role for top-down control of attention, we used a feature-based search task with the target identity defined by its categorical feature. Critically, the search arrays consisted of two physically salient items that disabled the use of singleton detection mode. These settings encouraged cognitive control to play a role in the adjustment of selective attention (Bacon and Egeth, 1994). In support of this notion, a physically salient distractor that consistently captured attention in a singleton-based visual search task became actively suppressed when switching the task to a featurebased search (Gaspelin et al., 2015). Therefore, the distinction in the search mode could also be a main source of these seemingly contradictory findings.

Neurophysiology and functional brain imaging studies related to perceptual competition among multiple stimuli have suggested that there is mutual suppression of the neural responses at the level of the receptive field (Moran and Desimone, 1985; Kastner et al., 1998; Reynolds et al., 1999) and feature-based suppression across the whole visual field (Moher et al., 2014; Störmer and Alvarez, 2014). Meanwhile, theories of attention suggest that biased activation due to the allocation of attention starts as early as in V1 if more than one item competes for priority in the visual field (Motter, 1993; Ito and Gilbert, 1999). In the present study, we took advantage of the forward encoding model with its assumption about the underlying relation between the orientation-tuned neuronal populations and the measured fMRI signals. With this model, we were able to detect the population-level neuronal changes specific to the reward-associated orientations in V1. These results provided clear evidence for featurebased competition between retinal locations at V1. Furthermore, our results are also consistent with the previous studies that reported attentional suppressive effects in V1 and subcortical areas (Gouws et al., 2014). Critically, our findings suggest that the weakened channel responses to the distractor with reward salience were correlated with reduced behavioral costs in search performance, confirming the central roles of reward salience and target-distractor competition in attentional suppression.

Substantial evidence suggests the important role of the midbrain dopamine neurons in stimulus-reward associative learning (Schultz et al., 1997). Our finding of the patterns for midbrain activity in VTA and the channel response in V1 suggests that greater midbrain activation led to stronger suppression in sensory areas. The possible account for such a VTA-V1 relationship could be drawn from the literature that has suggested the central role of reward-related signals in mediating higher-order cognitive functions (Frank et al., 2001). In the high reward distractor and low target salience condition, our observations of stronger activity in VTA may enhance the dopaminergic projection to the prefrontal cortex, enabling strengthened top-down control of attention effect in visual areas (Noudoost and Moore, 2011). Thus, the weakened sensory representation of the reward salient distractor as reflected in V1 channel responses could be ascribed to the increased top-down suppression triggered by midbrain modulation. This interpretation also parallels with the findings of reward effects in V1 activity in rats (Shuler and Bear, 2006), monkeys (Stănişor et al., 2013), and humans (Serences, 2008). Nevertheless, the present data were not sufficient to draw a direct inference between the patterns of activity in VTA and channel responses in V1. Further studies with specialized designs are required to verify this account. 
The recent findings of automatic attraction of attention by reward-associated items (Della Libera and Chelazzi, 2009; Peck et al., 2009; Raymond and O'Brien, 2009; Anderson et al., 2011a; Hickey and van Zoest, 2012) are in accordance with the classical idea in learning theory that the stimulus with high predictiveness of reward or important events receives attentional priority (Mackintosh, 1975; Le Pelley et al., 2016). This principle of associative learning has been suggested to play important roles in shaping multiple cognitive functions, such as working memory (Gong and Li, 2014; Wallis et al., 2015; Thomas et al., 2016) and long-term memory (Murayama and Kitagami, 2014). Moreover, it has been demonstrated that, by holding reward-associated cues in working memory, cognitive control could be selectively modulated by reward to enhance distractor suppression accompanied with the increased amplitude of the Pd component (Sawaki et al., 2015) or frontal theta oscillation (Gong et al., 2016). In a closely related fMRI study, reward was associated with a specific category in naturalistic scenes (Hickey and Peelen, 2015). Using the multivoxel pattern analysis approach, they found decreased information for scenes with reward-related distractors compared with reward-neutral distractors in the object-selective visual cortex. Their study and ours shared key findings in demonstrating neural suppression in the visual cortex of reward salient distractors and the reward-driven changes in midbrain activity. Nevertheless, our results differed from those of Hickey and Peelen (2015) in two main aspects. First, whereas they did not directly control the relative physical salience between the target and distractor, our study included this factor and found a novel result that suggests a perceptual competition-dependent effect of suppression of reward-associated distractors. Second, Hickey and Peelen (2015) demonstrated the reduced behavioral cost accompanied with the suppressed information representation of distractors in the object-selective cortex. Our study, using the lower level visual feature of orientation, showed that the behavioral benefit of high reward versus low reward-associated distractors was realized at the initial stage of visual processing in V1. These two studies complement each other and suggest the implementation of suppression of reward salience in early visual pathways. To our knowledge, the results from the present study demonstrate, for the first time, the weakened representation of reward-associated distractors in $\mathrm{V} 1$ through enhanced suppression.

\section{References}

Anderson BA, Laurent PA, Yantis S (2014) Value-driven attentional priority signals in human basal ganglia and visual cortex. Brain Res 1587:88-96. CrossRef Medline

Anderson BA, Laurent PA, Yantis S (2011a) Value-driven attentional capture. Proc Natl Acad Sci U S A 108:10367-10371. CrossRef Medline

Anderson BA, Laurent PA, Yantis S (2011b) Learned value magnifies salience-based attentional capture. PLoS One 6:e27926. CrossRef Medline

Anderson BA, Kuwabara H, Wong DF, Gean EG, Rahmim A, Brašić JR, George N, Frolov B, Courtney SM, Yantis S (2016) The role of dopamine in value-based attentional orienting. Curr Biol 26:550-555. CrossRef Medline

Awh E, Belopolsky AV, Theeuwes J (2012) Top-down versus bottom-up attentional control: a failed theoretical dichotomy. Trends Cogn Sci 16: 437-443. CrossRef Medline

Bacon WF, Egeth HE (1994) Overriding stimulus-driven attentional capture. Percept Psychophys 55:485-496. CrossRef Medline

Ballard IC, Murty VP, Carter RM, MacInnes JJ, Huettel SA, Adcock RA (2011) Dorsolateral prefrontal cortex drives mesolimbic dopaminergic regions to initiate motivated behavior. J Neurosci 31:10340-10346. CrossRef Medline

Bisley JW, Goldberg ME (2010) Attention, intention, and priority in the parietal lobe. Annu Rev Neurosci 33:1-21. CrossRef Medline

Brouwer GJ, Heeger DJ (2009) Decoding and reconstructing color from responses in human visual cortex. J Neurosci 29:13992-14003. CrossRef Medline

Brouwer GJ, Heeger DJ (2011) Cross-orientation suppression in human visual cortex. J Neurophysiol 106:2108-2119. CrossRef Medline

Buracas GT, Boynton GM (2002) Efficient design of event-related fMRI experiments using M-sequences. Neuroimage 16:801-813. CrossRef Medline

Chelazzi L, Eštočinová J, Calletti R, Lo Gerfo E, Sani I, Della Libera C, Santandrea E (2014) Altering spatial priority maps via reward-based learning. J Neurosci 34:8594-8604. CrossRef Medline

Dale M (1999) Optimal experimental design for event-related fMRI. Hum Brain Mapp 8:109-114. CrossRef Medline

Della Libera C, Chelazzi L (2009) Learning to attend and to ignore is a matter of gains and losses. Psychol Sci 20:778-784. CrossRef Medline

Desimone R, Duncan J (1995) Neural mechanisms of selective visual attention. Annu Rev Neurosci 18:193-222. CrossRef Medline

Eapen M, Zald DH, Gatenby JC, Ding Z, Gore JC (2011) Using highresolution MR imaging at $7 \mathrm{~T}$ to evaluate the anatomy of the midbrain dopaminergic system. AJNR Am J Neuroradiol 32:688-694. CrossRef Medline

Egeth HE, Yantis S (1997) Visual attention: control, representation, and time course. Annu Rev Psychol 48:269-297. CrossRef Medline

Engel SA, Glover GH, Wandell BA (1997) Retinotopic organization in human visual cortex and the spatial precision of functional MRI. Cereb Cortex 7:181-192. CrossRef Medline

Failing M, Nissens T, Pearson D, Le Pelley M, Theeuwes J (2015) Oculomotor capture by stimuli that signal the availability of reward. J Neurophysiol 114:2316-2327. CrossRef Medline

Fecteau JH, Munoz DP (2006) Salience, relevance, and firing: a priority map for target selection. Trends Cogn Sci 10:382-390. CrossRef Medline

Frank MJ, Loughry B, O'Reilly RC (2001) Interactions between frontal cortex and basal ganglia in working memory: a computational model. Cogn Affect Behav Neurosci 1:137-160. CrossRef Medline

Gaspar JM, McDonald JJ (2014) Suppression of salient objects prevents distraction in visual search. J Neurosci 34:5658-5666. CrossRef Medline

Gaspar JM, Christie GJ, Prime DJ, Jolicœur P, McDonald JJ (2016) Inability to suppress salient distractors predicts low visual working memory capacity. Proc Natl Acad Sci U S A 113:3693-3698. CrossRef Medline

Gaspelin N, Leonard CJ, Luck SJ (2015) Direct evidence for active suppression of salient-but-irrelevant sensory inputs. Psychol Sci 26:1740-1750. CrossRef Medline

Geng JJ, Diquattro NE (2010) Attentional capture by a perceptually salient non-target facilitates target processing through inhibition and rapid rejection. J Vis 10:5. CrossRef Medline

Gong M, Li S (2014) Learned reward association improves visual working memory. J Exp Psychol Hum Percept Perform 40:841-856. CrossRef Medline

Gong M, Yang F, Li S (2016) Reward association facilitates distractor suppression in human visual search. Eur J Neurosci 43:942-953. CrossRef Medline

Gouws AD, Alvarez I, Watson DM, Uesaki M, Rodgers J, Morland AB (2014) On the role of suppression in spatial attention: evidence from negative BOLD in human subcortical and cortical structures. J Neurosci 34:1034710360. CrossRef Medline

Hickey C, Peelen MV (2015) Neural mechanisms of incentive salience in naturalistic human vision. Neuron 85:512-518. CrossRef Medline

Hickey C, Chelazzi L, Theeuwes J (2010) Reward changes salience in human vision via the anterior cingulate. J Neurosci 30:11096-11103. CrossRef Medline

Hickey C, Chelazzi L, Theeuwes J (2011) Reward has a residual impact on target selection in visual search, but not on the suppression of distractors. Vis Cogn 19:117-128. CrossRef

Hickey C, van Zoest W (2012) Reward creates oculomotor salience. Curr Biol 22:R219-R220. CrossRef Medline

Houghton G, Tipper SP (1994) A model of inhibitory mechanisms in selective attention. In: Inhibitory processes in attention, memory, and language, pp 53-112. San Diego: Academic.

Houghton G, Tipper SP, Weaver B, Shore DI (1996) Inhibition and interference in selective attention: some tests of a neural network model. Vis Cogn 3:119-164. CrossRef

Ito M, Gilbert CD (1999) Attention modulates contextual influence in the 
primary visual cortex of alert monkeys. Neuron 22:593-604. CrossRef Medline

Kastner S, De Weerd P, Desimone R, Ungerleider LG (1998) Mechanisms of directed attention in the human extrastriate cortex as revealed by functional MRI. Science 282:108-111. CrossRef Medline

Kastner S, Ungerleider LG (2001) The neural basis of biased competition in human visual cortex. Neuropsychologia 39:1263-1276. CrossRef Medline

Laurent PA, Hall MG Anderson BA and Yantis S (2015) Valuable orientations capture attention. Vis Cognition 23(1-2):133-146.

Le Pelley ME, Mitchell CJ, Beesley T, George DN, Wills AJ (2016) Attention and associative learning in humans: an integrative review. Psychol Bull 142:1111-1140. CrossRef Medline

Lee J, Shomstein S (2014) Reward-based transfer from bottom-up to topdown search tasks. Psychol Sci 25:466-475. CrossRef Medline

Le Pelley ME, Pearson D, Griffiths O, Beesley T (2015) When goals conflict with values: counterproductive attentional and oculomotor capture by reward-related stimuli predictiveness-driven attentional capture. J Exp Psychol Gen 144:158-171. CrossRef Medline

Mackintosh NJ (1975) A theory of attention: variations in the associability of stimuli with reinforcement. Psychol Rev 82:276-298. CrossRef

Martinez-Trujillo JC, Treue S (2004) Feature-based attention increases the selectivity of population responses in primate visual cortex. Curr Biol 14:744-751. CrossRef Medline

Moher J, Lakshmanan BM, Egeth HE, Ewen JB (2014) Inhibition drives early feature-based attention. Psychol Sci 25:315-324. CrossRef Medline

Moran J, Desimone R (1985) Selective attention gates visual processing in the extrastriate cortex. Science 229:782-784. CrossRef Medline

Motter BC (1993) Focal attention produces spatially selective processing in visual cortical areas V1, V2, and V4 in the presence of competing stimuli. J Neurophysiol 70:909-919. Medline

Murayama K, Kitagami S (2014) Consolidation power of extrinsic rewards: reward cues enhance long-term memory for irrelevant past events. J Exp Psychol Gen 143:15-20. CrossRef Medline

Murty VP, Shermohammed M, Smith DV, Carter RM, Huettel SA, Adcock RA (2014) Resting state networks distinguish human ventral tegmental area from substantia nigra. Neuroimage 100:580-589. CrossRef Medline

Noudoost B, Moore T (2011) Control of visual cortical signals by prefrontal dopamine. Nature 474:372-375. CrossRef Medline

Peck CJ, Jangraw DC, Suzuki M, Efem R, Gottlieb J (2009) Reward modulates attention independently of action value in posterior parietal cortex. J Neurosci 29:11182-11191. CrossRef Medline

Ptak R (2012) The frontoparietal attention network of the human brain: action, saliency, and a priority map of the environment. Neuroscientist 18:502-515. CrossRef Medline

Rajsic J, Perera H, Pratt J (2017) Learned value and object perception: Accelerated perception or biased decisions?. Atten Percept Psychophys 79: 603-613. CrossRef Medline

Raymond JE, O’Brien JL (2009) Selective visual attention and motivation: The consequences of value learning in an attentional blink task. Psychol Sci 20:981-988. CrossRef Medline

Reynolds JH, Chelazzi L, Desimone R (1999) Competitive mechanisms sub- serve attention in macaque areas V2 and V4. J Neurosci 19:1736-1753. Medline

Sawaki R, Luck SJ (2010) Capture versus suppression of attention by salient singletons: electrophysiological evidence for an automatic attend-to-me signal. Atten Percept Psychophys 72:1455-1470. CrossRef Medline

Sawaki R, Luck SJ (2011) Active suppression of distractors that match the contents of visual working memory. Vis Cogn 19:956-972. CrossRef Medline

Sawaki R, Geng JJ, Luck SJ (2012) A common neural mechanism for preventing and terminating the allocation of attention. J Neurosci 32:1072510736. CrossRef Medline

Sawaki R, Luck SJ, Raymond JE (2015) How attention changes in response to incentives. J Cogn Neurosci 27:2229-2239. CrossRef Medline

Schiller PH, Finlay BL, Volman SF (1976) Quantitative studies of single-cell properties in monkey striate cortex: III. Spatial frequency. J Neurophysiol 39:1334-1351. Medline

Schultz W, Dayan P, Montague PR (1997) A neural substrate of prediction and reward. Science 275:1593-1599. CrossRef Medline

Serences JT (2008) Value-based modulations in human visual cortex. Neuron 60:1169-1181. CrossRef Medline

Serences JT, Saproo S (2012) Computational advances towards linking BOLD and behavior. Neuropsychologia 50:435-446. CrossRef Medline

Sereno MI, Dale AM, Reppas JB, Kwong KK, Belliveau JW, Brady TJ, Rosen BR, Tootell RB (1995) Borders of multiple visual areas in humans revealed by functional magnetic resonance imaging. Science 268:889-893. CrossRef Medline

Sha LZ, Jiang YV (2016) Components of reward-driven attentional capture. Atten Percept Psychophys 78:403-414. CrossRef Medline

Shuler MG, Bear MF (2006) Reward timing in the primary visual cortex. Science 311:1606-1609. CrossRef Medline

Stănişor L, van der Togt C, Pennartz CM, Roelfsema PR (2013) A unified selection signal for attention and reward in primary visual cortex. Proc Natl Acad Sci U S A 110:9136-9141. CrossRef Medline

Störmer VS, Alvarez GA (2014) Feature-based attention elicits surround suppression in feature space. Curr Biol 24:1985-1988. CrossRef Medline

Swindale NV (1998) Orientation tuning curves: empirical description and estimation of parameters. Biol Cybern 56:45-56. CrossRef Medline

Theeuwes J (1992) Perceptual selectivity for color and form. Percept Psychophys 51:599-606. CrossRef Medline

Thomas PM, FitzGibbon L, Raymond JE (2016) Value conditioning modulates visual working memory processes. J Exp Psychol Hum Percept Perform 42:6-10. CrossRef Medline

van Schouwenburg M, Aarts E, Cools R (2010) Dopaminergic modulation of cognitive control: distinct roles for the prefrontal cortex and the basal ganglia. Curr Pharm Des 16:2026-2032. CrossRef Medline

Wallis G, Stokes MG, Arnold C, Nobre AC (2015) Reward boosts working memory encoding over a brief temporal window. Vis Cogn 23:291-312. CrossRef

Weaver MD, van Zoest W, Hickey C (2017) A temporal dependency account of attentional inhibition in oculomotor control. Neuroimage 147: 880-894. CrossRef Medline 